\title{
INVISCID AND VISCOUS MODELS OF AXISYMMETRIC FLUID JETS OR PLUMES
}

\author{
NICHOLAS A. LETCHFORD ${ }^{1}$, LAWRENCE K. FORBES ${ }^{\bowtie 1}$ and GRAEME C. HOCKING ${ }^{2}$
}

(Received 5 May, 2012; revised 3 September, 2012)

\begin{abstract}
The vertical rise of a round plume of light fluid through a surrounding heavier fluid is considered. An inviscid model is analysed in which the boundary of the plume is taken to be a sharp interface. An efficient spectral method is used to solve this nonlinear freeboundary problem, and shows that the plume narrows as it rises. A generalized condition is also introduced at the boundary, and allows the ambient fluid to be entrained into the rising plume. In this case, the fluid plume first narrows then widens as it rises. These features are confirmed by an asymptotic analysis. A viscous model of the same situation is also proposed, based on a Boussinesq approximation. It qualitatively confirms the widening of the plume due to entrainment of the ambient fluid, but also shows the presence of vortex rings around the interface of the rising plume.
\end{abstract}

2010 Mathematics subject classification: 76D25.

Keywords and phrases: fluid jet, axisymmetric flow, inviscid theory, spectral methods, plume, Boussinesq approximation, entrainment.

\section{Introduction}

In a plume, a stream of light fluid rises through an ambient fluid of greater density. There is an extremely extensive literature on plumes and jets (or fountains), and they have applications to a wide variety of situations. The review by Woods [33] describes a range of natural processes in which turbulent buoyant plumes occur, including volcanic eruptions. They are also of importance in a number of astrophysical situations, and Reynolds et al. [24] present numerical calculations of bipolar plumes generated on galactic length scales. A remarkable example of plumes occurs from volcanic vents deep on the ocean floor; these "black smokers" are discussed by Wilcock [32] and are often associated with communities of thermophilic bacteria [23].

\footnotetext{
${ }^{1}$ School of Mathematics and Physics, University of Tasmania, Private Bag 37, Hobart, Tasmania 7001, Australia; e-mail: Larry.Forbes@utas.edu.au.

${ }^{2}$ School of Chemical and Mathematical Sciences, Murdoch University, 90 South Street, Murdoch, Western Australia 6150, Australia; e-mail: G.Hocking@murdoch.edu.au.

(C) Australian Mathematical Society 2012, Serial-fee code 1446-1811/2012\$16.00
} 
Possibly the simplest mathematical models of rising plumes involve inviscid fluids with a sharp free surface or interface. Such a model was proposed by Geer and Strikwerda [13], although they considered a plume falling downwards under gravity; the plume speeds up and narrows as it falls, and the authors analysed the flow with a perturbation technique based on the narrowness of the plume. Their model allowed for plumes of arbitrary cross section, and is essentially equivalent to an inviscid plume of light fluid rising through a heavier fluid under reduced gravity, due to its natural buoyancy. Tuck [28] likewise considered an inviscid jet, which was annular in cross section and could be directed either upwards or downwards. Since the jet was denser than the surrounding fluid, when directed upwards it became a fountain, and under gravity it rose to a limiting height dictated by the fluid velocity at the nozzle.

Planar inviscid fluid jets have also been studied, and an analysis in a similar spirit to Tuck's [28] work was carried out by Borgas and Tuck [4] assuming a slender two-dimensional jet. Christodoulides and Dias [7] used complex-variable theory and conformal mapping methods to solve for the shape of a vertically directed planar fountain that strikes a horizontal plate before spreading laterally and then falling back down under gravity. (The corresponding axisymmetric flow, involving a "water bell" beneath a horizontal plate, has been investigated theoretically and confirmed in a series of elegant experiments by Button et al. [5].) Similar methods to those of Christodoulides and Dias [7] were employed by Hocking and Forbes [14] in an analysis of a planar buoyant plume that rises vertically before spreading horizontally across an upper ceiling; plumes of this type occur in building fires. Their solutions were independent of time, and consequently only existed for flow speeds at the nozzle above some threshold minimum speed, and at that minimum configuration the plume interface formed a stagnation point at the nozzle itself. Recently, Forbes and Hocking [12] considered a similar planar inviscid plume, and allowed the fluid to be emitted either vertically or at an angle from the nozzle at the bottom of the fluid layer. Their paper was concerned with time-dependent effects in the plume, and showed that when oriented vertically, the unsteady inviscid interface tended closely to the steady profile predicted by a slender-plume asymptotic theory, as time increased. However, when the plume entered the fluid layer at any other angle, it was unstable, and consequently no steady-state configuration was achieved, even though the asymptotic analysis predicted such a possible outcome. Numerically, it was found that the nonvertical plume ultimately developed points of very high curvature on the plume interface, and these were responsible for the failure of the numerical solution method. It was suggested that the interface develops curvature singularities within finite time, similar to those predicted by Moore [20] in the evolution of the KelvinHelmholtz instability. Forbes and Hocking [12] also included viscous effects in the same problem, and found that there was then no qualitative difference between vertical and nonvertical planar plumes, since neither converged to a steady-state configuration as time increased, but rather each was fundamentally unsteady due to the presence of entrainment vortices formed along the interface. 
In nature, all plumes are of course affected by viscosity, and the flow is in fact often turbulent. This poses a severe problem for computer modelling of such phenomena. Kaminski et al. [17] considered an axisymmetric turbulent plume and developed a system of equations governing certain bulk averaged turbulent quantities, and these were used to quantify turbulent entrainment in experimental studies of a negatively buoyant plume. Plumes of this type occur in outflows from volcanos. Scase et al. [25] used a large-eddy turbulent code to simulate plumes in which the momentum strength is increased rapidly, and a truly large-scale computational study of a fully developed three-dimensional plume was undertaken by Plourde et al. [22], using direct numerical simulation with grid sizes of the order of the Kolmogorov length scale of the turbulence itself. Lin and Armfield [18] similarly used direct numerical simulation, although for a plume that is axisymmetric, for which the Boussinesq approximation has been made, and in the transition region between purely laminar and fully turbulent flow conditions. Nevertheless, simulating turbulent plumes using averaged momentum equations with a "closure" model for the turbulence is not without difficulty, and Bhat and Krothapalli [3] argued that the results obtained are very sensitive to the form of the closure assumption chosen. In a recent investigation of four integral models of turbulent plumes, Scase and Hewitt [26] similarly observed that none of the models are entirely adequate, in part due to ill-conditioning associated with the unbounded growth of small-wavelength disturbances.

In the "classical" plume model of Morton et al. [21], hereafter referred to as MTT, the flow is regarded as fully turbulent, and the Reynolds number is effectively taken to be infinite in the governing equations. The outflow nozzle is approximated to be a point source, so that similarity arguments can be applied to the spreading plume. A Boussinesq approximation is also made, in which local variations in the fluid density are taken to be small in comparison to the mean density. Crucial to this theory is an entrainment assumption; it makes the hypothesis that the radial and vertical components of the fluid velocity, $u$ and $w$, are in some sense statistical averages of the fluctuating turbulent quantities, and that there is a mean radial component $u$ of fluid motion at the edge of the plume that is proportional to the vertical speed $w$ within the plume. At the edge, there is thus a relationship of the form

$$
u=\alpha w,
$$

in which $\alpha$ is an entrainment coefficient, assumed constant. This assumption (1.1) is discussed further by Turner [29, 30], who argues that the numerical value of $\alpha$ is of the order of 0.1 , although it depends on the assumed profile of the axial component $w$, and so has different values for a Gaussian or a "top-hat" profile. Further detailed discussion of the model (1.1) is given in review articles by List [19] and Hunt and van den Bremer [15], who indicate the success of the MTT entrainment model in describing turbulent plumes. Hunt and Kaye [16] additionally classify turbulent plumes as "lazy", "pure" or "forced", depending on a combination of their source volume flux, momentum flux and buoyancy flux, and they likewise conclude that the MTT assumption (1.1) works well for "lazy" plumes, which have only weak 
momentum flux. In that case, they find that the plume narrows as it leaves the nozzle, before eventually widening as a result of entrainment of the ambient fluid into the plume. Consequently, the lazy plume exhibits a minimum radius at some height above the nozzle.

The purpose of the present paper is to study the time-dependent evolution of an axisymmetric plume or jet. Following Lin and Armfield [18], we do not focus on the turbulent regime, but rather address the transition region in which plume speeds and Reynolds numbers lie between laminar and turbulent behaviour. To begin, a purely inviscid solution is developed, following a similar philosophy to that of Tuck [28]. Laplace's equation for a velocity potential is solved within the plume, subject to nonlinear boundary conditions at the plume interface. We also propose a modification to the traditional kinematic boundary condition that allows for entrainment of the outer ambient fluid into the plume, while essentially retaining an inviscid formulation of the problem. This is done using a generalized condition based on the entrainment approximation (1.1) of MTT [21]; however, there is no assumption of turbulent behaviour generally within the fluid, and the condition only holds on the interface. This is described in Section 2. The justification for this new nonturbulent entrainment condition is based on the observation that in a number of viscous interfacial flow problems, such as the recent study of the Rayleigh-Taylor instability by Forbes [10] and the analysis of the Kelvin-Helmholtz instability by Chen and Forbes [6], it is observed that the vorticity is essentially zero throughout the fluid, except in a narrow layer near the interface. Consequently, the fluid behaves as if it were inviscid, except near the interface, at which entrainment can occur. Nevertheless, this is an approximation, and cannot generate vorticity beyond the interface. This unsteady inviscid problem is formulated using the spectral method of Forbes et al. [11] in Section 3, for the conventional plume as well as for the case in which entrainment occurs. An asymptotic analysis is presented for both cases in Section 4.

Similarly to the case of the planar plume studied by Forbes and Hocking [12], we examine the effect of viscosity in the present situation, using a Boussinesq approximation, and compare it qualitatively with the results of the inviscid models. The formulation and its spectral solution are outlined in Section 5, and the results are discussed in Section 6. A discussion in Section 7 concludes the paper.

\section{The inviscid model}

Consider an ideal fluid of density $\rho_{2}$ contained within a horizontal layer. A cylindrical coordinate system is located with its origin at the centre of the nozzle and its $z$-axis pointing vertically, opposing the direction of the gravitational acceleration $-g \mathbf{e}_{z}$. The fluid is bounded below at $z=0$ and above at $z=H$. A circular nozzle of radius $A$ is centred at $r=0$ and emits a source fluid of lighter density $\rho_{1}<\rho_{2}$. At the nozzle, the velocity vector of the source fluid is oriented purely vertically with magnitude $V$. It rises under positive buoyancy through the heavier ambient fluid, and for simplicity is assumed to be absorbed into a permeable material at the upper boundary $z=H$. 


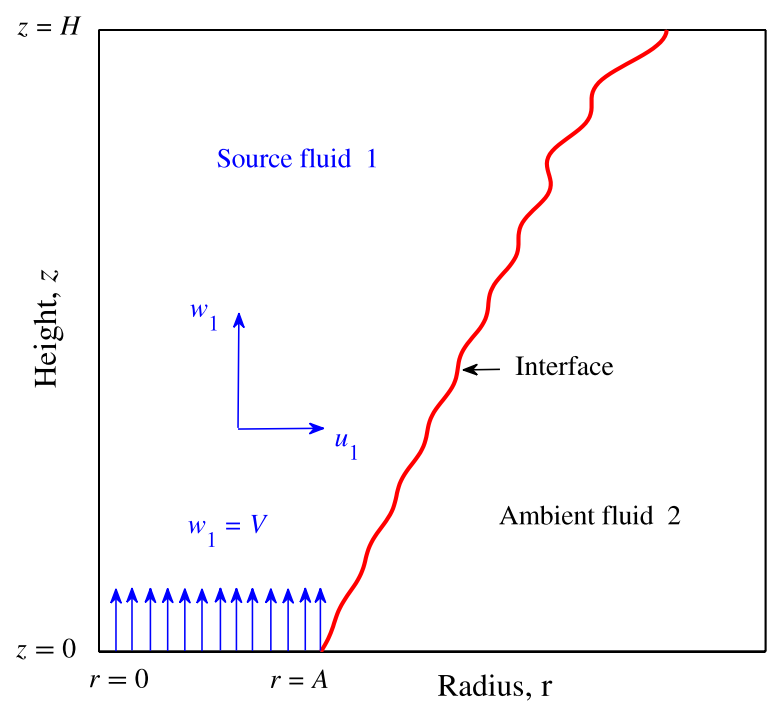

FIGURE 1. A schematic diagram of the dimensional flow for the inviscid plume, showing the two fluids separated by an interface.

The plume is assumed to remain axisymmetric, so that there is no azimuthal velocity component and no dependence on the angular coordinate $\theta$. The velocity vector within the plume is expressed as $\mathbf{q}_{1}=u_{1} \mathbf{e}_{r}+w_{1} \mathbf{e}_{z}$ and has components $u_{1}$ and $w_{1}$ in the radial and axial directions. A sketch of the flow configuration in these dimensional variables is given in Figure 1.

Nondimensional variables are introduced forthwith, and unless otherwise specified are used from now on. All lengths are scaled with respect to the radius of the nozzle $A$, and velocities are scaled with respect to the speed $V$ of the source fluid. Time is measured relative to the quantity $A / V$ and pressure relative to $\rho_{1} g A$. This inviscid problem is thus dependent upon the dimensionless parameters

$$
h=\frac{H}{A}, \quad D=\frac{\rho_{2}}{\rho_{1}}, \quad F=\frac{V}{\sqrt{g A}} .
$$

The constant $h$ is the nondimensional plume height, $D$ is the density ratio $(D>1)$, and $F$ is the Froude number which gives a measure of the fluid speed at the nozzle.

Inviscid fluids are not capable of supporting internal shear stress, and so the outer ambient fluid is assumed to be motionless, with velocity $\mathbf{q}_{2}=\mathbf{0}$. The pressure in the ambient fluid is therefore hydrostatic, with $P_{2}=p_{A}-D z$, in which $p_{A}$ is a dimensionless reference pressure on the nozzle, at location $(r, z)=(1,0)$. The inner source fluid flows irrotationally, and so a velocity potential $\Phi_{1}$ may be defined there, in terms of which the velocity vector can be written $\mathbf{q}_{1}=\nabla \Phi_{1}$. The source fluid is separated from the outer ambient fluid by an interface $r=R(z, t)$ that emerges from the location $(r, z)=(1,0)$ at the nozzle. 
As both fluids are incompressible, the velocity potential $\Phi_{1}$ for the moving inner fluid satisfies Laplace's equation

$$
\frac{1}{r} \frac{\partial}{\partial r}\left(r \frac{\partial \Phi_{1}}{\partial r}\right)+\frac{\partial^{2} \Phi_{1}}{\partial z^{2}}=0 \quad \text { in } 0<r<R(z, t), 0<z<h .
$$

On the bottom $z=0$, the vertical velocity component $w$ satisfies the boundary condition

$$
w(r, 0, t)= \begin{cases}1 & \text { if } 0<r<1 \\ 0 & \text { if } r>1\end{cases}
$$

At the top of the fluid region, on the plane $z=h$, the plume is assumed to be absorbed vertically into the surface, for simplicity. This might correspond to a permeable boundary, such as a horsehair mat, being present at the upper surface. This is represented by the condition

$$
\left.\frac{\partial w_{1}}{\partial z}\right|_{z=h}=0
$$

These conditions (2.3) and (2.4) allow the solution to be developed using a spectral technique, as is discussed later. In their model of a planar plume, Hocking and Forbes [14] assumed a rigid upper boundary at $z=h$, and a similar condition could be considered here, too. Condition (2.4) has been adopted for simplicity, however, as it avoids the additional complication of a horizontal outflow along the upper boundary.

There is also a kinematic condition to be satisfied on the interface $r=R(z, t)$. This normally states that the there can be no flow normal to the interface, so that $\mathbf{q}_{1} \cdot \mathbf{n}=0$ there. Here $\mathbf{n}$ denotes the unit normal vector at the surface of the plume. As explained in Section 1, we also wish to mimic the effect of nonturbulent entrainment of the ambient fluid into the moving plume, by allowing flow normal to the interface, with speed proportional to the tangential component of the fluid within the plume. This is based on the MTT model (1.1) for turbulent fluid [21], but here is only taken to apply locally at the interface. We assume that $\mathbf{q}_{1} \cdot \mathbf{n}=k\left|\mathbf{q}_{1} \cdot \tau\right|$, in which $\tau$ represents the unit vector tangential to the interface. The constant $k$ is the nonturbulent entrainment coefficient, and plays an analogous role to the turbulent constant $\alpha$ in equation (1.1). In this approximation, it is assumed that the proportion $k$ of ambient fluid entrained is sufficiently small that the changes to the density within the plume can be ignored. Thus

$$
\frac{D \mathcal{H} / D t}{\|\nabla \mathcal{H}\|}=k\left|\mathbf{q}_{1} \cdot \tau\right| \quad \text { on } r=R(z, t),
$$

where the equation of the interface is written as $\mathcal{H}(r, z, t)=r-R(z, t)=0$. Evaluating equation (2.5) leads to

$$
\frac{\partial R}{\partial t}=u_{1}-w_{1} \frac{\partial R}{\partial z}+k\left|u_{1} \frac{\partial R}{\partial z}+w_{1}\right| \quad \text { on } r=R(z, t) .
$$

Notice that, when $k=0$, the conventional nonentraining kinematic boundary condition is recovered. The work of Turner [29, 30] and Kaminski et al. [17] suggests that reasonable values of $k$ lie in the approximate interval $0.07<k<0.16$. 
There is also a dynamic condition to be imposed at the interface between the two fluids. It states that the pressures must be equal there, and from Bernoulli's equation in each fluid [2], this gives

$$
\frac{\partial \Phi_{1}}{\partial t}+\frac{1}{2}\left(u_{1}^{2}+w_{1}^{2}\right)+\frac{1-D}{F^{2}} z=\frac{1}{2}
$$

on the interface $r=R(z, t)$.

\section{Solution method for the inviscid plume}

The time-dependent nonlinear inviscid problem in Section 2 is solved using the method proposed by Forbes et al. [11], which is similar to the spectral approach of Faltinsen et al. [8]. This technique was used by Forbes and Hocking [12] to model a planar plume, in the inviscid case, and the present problem is an extension of that work to axisymmetric geometry. Here, the velocity potential for the inner source fluid is given by

$$
\Phi_{1}(r, z, t)=z+C_{0}(t)+\sum_{n=1}^{N} C_{n}(t) \frac{\mathrm{I}_{0}\left(\alpha_{n} r\right)}{\mathrm{I}_{0}\left(\alpha_{n}\right)} \cos \left(\alpha_{n} z\right)
$$

in which

$$
\alpha_{n}=\frac{2 n-1}{2 h} .
$$

This form (3.1) with constants (3.2) satisfies Laplace's equation (2.2), the lower boundary condition (2.3) and the top boundary condition (2.4). The corresponding streamfunction is expressed as

$$
\psi(r, z, t)=\frac{1}{2} r-\sum_{n=1}^{N} C_{n}(t) \frac{\mathrm{I}_{1}\left(\alpha_{n} r\right)}{\mathrm{I}_{0}\left(\alpha_{n}\right)} \sin \left(\alpha_{n} z\right) .
$$

In expressions (3.1) and (3.3), the functions $I_{0}$ and $I_{1}$ are the modified Bessel functions of the first kind, of orders zero and one, respectively [1, p. 374]. For steady flow, it may be shown that the product $r \psi$ is constant along a streamline in cylindrical coordinates. The interface $R$ is represented by the Fourier series

$$
R(z, t)=1+\sum_{n=1}^{N} B_{n}(t) \sin \left(\alpha_{n} z\right)
$$

The three Fourier representations (3.1), (3.3) and (3.4) all involve a finite number $N$ of terms, but become exact as $N \rightarrow \infty$.

The two remaining boundary conditions (2.6) and (2.7) on the interface are used to solve for the coefficients $C_{0}(t), C_{n}(t)$ and $B_{n}(t)$. Following Forbes et al. [11], 
the radial and vertical velocity components are first evaluated directly at the free surface $r=R(z, t)$, to give

$$
\begin{aligned}
& U_{1}(z, t)=\sum_{n=1}^{N} \alpha_{n} C_{n}(t) \frac{\mathrm{I}_{1}\left(\alpha_{n} R\right)}{\mathrm{I}_{0}\left(\alpha_{n}\right)} \cos \left(\alpha_{n} z\right), \\
& W_{1}(z, t)=1-\sum_{n=1}^{N} \alpha_{n} C_{n}(t) \frac{\mathrm{I}_{0}\left(\alpha_{n} R\right)}{\mathrm{I}_{0}\left(\alpha_{n}\right)} \sin \left(\alpha_{n} z\right),
\end{aligned}
$$

and these are used directly in the kinematic and dynamic boundary conditions.

The kinematic condition (2.6) is spectrally decomposed into its Fourier components by multiplying by the basis functions $\sin \left(\alpha_{\ell} z\right), \ell=1,2, \ldots, N$, and integrating over the interval $0<z<h$. This yields a system of ordinary differential equations for the coefficients $B_{\ell}$ in the form

$$
B_{\ell}^{\prime}(t)=\frac{2}{h} \int_{0}^{h}\left[J_{1}+K_{1}\right] \sin \left(\alpha_{\ell} z\right) d z, \quad \ell=1,2, \ldots, N .
$$

In (3.5) it has been convenient to define the intermediate quantities

$$
J_{1}=U_{1}-W_{1} \frac{\partial R}{\partial z}, \quad K_{1}=k\left|U_{1} \frac{\partial R}{\partial z}+W_{1}\right|,
$$

where $k$ is the nonturbulent entrainment coefficient.

A similar procedure is applied to the dynamic condition (2.7). The zeroth-order Fourier mode is obtained by integrating this boundary condition over the interval $0<z<h$, and gives the result

$$
h C_{0}(t)+\sum_{n=1}^{N} F_{0 n} C_{n}^{\prime}(t)=\frac{h}{2}+\frac{(D-1) h^{2}}{2 F^{2}}-\frac{1}{2} G_{0} .
$$

The higher-order Fourier decomposition of the dynamic condition is likewise derived by multiplying equation (2.7) by the basis functions $\cos \left(\alpha_{\ell} z\right), \ell=1,2, \ldots, N$, and integrating over $0<z<h$. After a little algebra, this produces the further system of ordinary differential equations

$$
\begin{aligned}
& -\frac{\cos (\ell \pi)}{\alpha_{\ell}} C_{0}^{\prime}(t)+\sum_{n=1}^{N} F_{\ell n} C_{n}^{\prime}(t) \\
& \quad=-\frac{\cos (\ell \pi)}{2 \alpha_{\ell}}-\frac{(D-1) h^{2}}{F^{2}}\left(\frac{h \cos (\ell \pi)}{\alpha_{\ell}}+\frac{1}{\alpha_{\ell}^{2}}\right)-\frac{1}{2} G_{\ell}, \quad \ell=1,2, \ldots, N .
\end{aligned}
$$

In equations (3.7) and (3.8) it has proved convenient to define further intermediate expressions

$$
\begin{aligned}
& F_{\ell n}(t)=\int_{0}^{h} \frac{\mathrm{I}_{0}\left(\alpha_{n} R\right)}{\mathrm{I}_{0}\left(\alpha_{n}\right)} \cos \left(\alpha_{\ell} z\right) \cos \left(\alpha_{n} z\right) d z, \\
& G_{\ell}(t)=\int_{0}^{h}\left(U_{1}^{2}+W_{1}^{2}\right) \cos \left(\alpha_{\ell} z\right) d z, \quad \ell=0,1, \ldots, N .
\end{aligned}
$$


The system of equations (3.5)-(3.8) represents a set of $2 N+1$ differential equations that are integrated forward in time using the classical fourth-order Runge-Kutta method. At each step in the integration, it is necessary to solve a matrix system of size $(N+1) \times(N+1)$ for the coefficients $C_{0}, C_{1}, \ldots, C_{N}$ using equations (3.7) and (3.8). The integrals involved in equation (3.5) and the intermediate quantities (3.9) are evaluated numerically using composite trapezoidal rule quadrature.

\section{Asymptotic solution for the inviscid plume}

In this section, an asymptotic solution to the inviscid system of equations in Section 2 is developed. The approximation is similar to classical shallow-water theory, as outlined by Stoker [27]. The plume is regarded as "slender", so that the pressure within it is approximately hydrostatic and only varies with height, according to the formula $p_{1} \approx P_{A}-D z$, in which $P_{A}$ represents the dimensionless pressure at $(r, z)=(1,0)$, at the edge of the nozzle on the tank bottom. In the outer ambient fluid, this hydrostatic relation holds exactly. As a consequence of this approximation, the vertical velocity component $w_{1}$ is taken to be independent of the radial coordinate $r$, and the radial component $u_{1}$ is small in comparison. The vertical component of Euler's momentum equation becomes approximately

$$
\frac{\partial w_{1}}{\partial t}+w_{1} \frac{\partial w_{1}}{\partial z} \approx \frac{D-1}{F^{2}} .
$$

The kinematic boundary condition (2.6) in this theory becomes

$$
u_{1} \approx \frac{\partial R}{\partial t}+w_{1} \frac{\partial R}{\partial z}-k w_{1} \quad \text { on } r=R(z, t) .
$$

The equation of mass conservation, $\operatorname{div} \mathbf{q}=0$, is integrated over the plume radius $0<r<R(z, t)$, and when the approximate condition (4.2) is employed, a second approximate equation is obtained, in the form

$$
\frac{\partial}{\partial t}\left(\frac{1}{2} R^{2}\right)+\frac{\partial}{\partial z}\left(\frac{1}{2} R^{2} w_{1}\right)-k R w_{1} \approx 0,
$$

in which $k$ is again the nonturbulent entrainment coefficient.

It is straightforward to obtain a steady-state solution to these asymptotic equations (4.1) and (4.3). The steady upward velocity component is at once found to be

$$
w_{1} \approx \sqrt{1+\frac{2(D-1) z}{F^{2}}} .
$$

Essentially this result is given by Geer and Strikwerda [13] and Tuck [28], although in variables that have been scaled differently than those used here.

Combining equations (4.3) and (4.4) yields a first-order linear differential equation for the steady interface profile $R(z)$. When there is no entrainment, the plume radius is 
found to decrease with height according to the relation

$$
R(z) \approx\left[1+\frac{2(D-1) z}{F^{2}}\right]^{-1 / 4}
$$

as is to be expected from conservation of mass and the fact that the vertical speed (4.4) increases with height. However, when entrainment is allowed, so that $k \neq 0$ in the present nonturbulent inviscid approximation (2.6), the asymptotic solution for the steady plume radius is derived in the form

$$
R(z) \approx \frac{2 k F^{2}}{5(D-1)}\left[1+\frac{2(D-1) z}{F^{2}}\right]+\left(1-\frac{2 k F^{2}}{5(D-1)}\right)\left[1+\frac{2(D-1) z}{F^{2}}\right]^{-1 / 4} .
$$

In this entraining case, equation (4.6) shows that the interface radius first decreases with height $z$ near the nozzle at $z=0$ for sufficiently small entrainment coefficient $k<(D-1) /\left(2 F^{2}\right)$. Eventually, however, the plume radius increases with height according to the behaviour $R(z) \sim(4 / 5) k z$ as $z \rightarrow \infty$, due to the additional fluid volume entrained by the rising plume. Thus, for small entrainment, there is a height at which the plume radius is at a minimum. This result is qualitatively similar to that obtained in the fully turbulent case by Hunt and Kaye [16].

It is, in fact, possible to solve the unsteady nonlinear equations (4.1) and (4.3) exactly, for suitable initial conditions, using the method of characteristics, at least in the nonentraining case $k=0$. The results are not presented here, but they show that the unsteady solution evolves to the steady solution (4.4), (4.5) by means of a pulse moving up the plume. These features are also seen in the fully nonlinear inviscid solution described in Section 3, and indicate that the steady-state asymptotic solution is stable in this approximate (inviscid) theory.

\section{The viscous Boussinesq model}

In this section, viscous effects on the plume are included, using a Boussinesq approximation to the full Navier-Stokes equations of viscous flow. This is based on the similar approach taken by Farrow and Hocking [9] to study the withdrawal of a two-layer fluid from a tank. The behaviour of the viscous plume is considerably more complicated than the purely inviscid case, particularly because the viscous fluid is capable of generating its own vorticity. As a result, ambient fluid will always be entrained into the plume, and in such a way as to involve rotational motion of the fluid.

Under the Boussinesq approximation, the density is regarded as a single continuous variable that may vary rapidly, yet smoothly, from the source fluid to the surrounding ambient fluid. Returning briefly to dimensional variables, the density can be expressed as $\rho(r, z, t)=\rho_{2}+\bar{\rho}(r, z, t)$. Here $\rho_{2}$ is the density of the ambient fluid far from the plume, and is assumed to be constant. The perturbation density $\bar{\rho}$ is assumed to be small in comparison with $\rho_{2}$, and the density ratio of the outer fluid to the inner fluid, $D=\rho_{2} / \rho_{1}$, is taken to be slightly larger than 1 . It is further assumed that the velocity 
of the fluid can also be represented by a single vector function that varies smoothly from the source to the ambient fluid.

The problem is nondimensionalized with respect to the same variables as in Section 2, using the radius of the nozzle $A$ as the reference length and the nozzle speed $V$ as the reference speed. Time is scaled with respect to the quantity $A / V$ and $\rho_{2}$ is taken as the reference density. The Boussinesq approximation makes the assumption that the continuity equation can effectively be "split" into an incompressible part,

$$
\frac{1}{r} \frac{\partial}{\partial r}(r u)+\frac{\partial w}{\partial z}=0
$$

and a separate transport equation for the density perturbation,

$$
\frac{\partial \bar{\rho}}{\partial t}+u \frac{\partial \bar{\rho}}{\partial r}+w \frac{\partial \bar{\rho}}{\partial z}=\sigma\left[\frac{1}{r} \frac{\partial}{\partial r}\left(r \frac{\partial \bar{\rho}}{\partial r}\right)+\frac{\partial^{2} \bar{\rho}}{\partial z^{2}}\right] .
$$

This second equation (5.2) can alternatively be derived from an energy equation, so that $\bar{\rho}$ may be regarded as a perturbation to the ambient temperature [18]. Here the constant $\sigma$ is a diffusion coefficient for density, or alternatively is the inverse of the product of the Reynolds number and Prandtl number if $\bar{\rho}$ is taken to be temperature.

Since the flow is rotational, a velocity potential can no longer be defined, as was possible for the inviscid case in Section 3. However, equation (5.1) nevertheless permits a streamfunction $\Psi$ to be defined through the relations

$$
u=-\frac{\partial \Psi}{\partial z}, \quad w=\frac{1}{r} \frac{\partial}{\partial r}(r \Psi)
$$

It is convenient to introduce vorticity, which is defined as the curl of the velocity vector $\mathbf{q}$, and only has a single component $\zeta$ in the azimuthal coordinate. This gives

$$
\zeta=\frac{\partial u}{\partial z}-\frac{\partial w}{\partial r}=-\nabla^{2} \Psi
$$

An equation for the vorticity $\zeta$ is obtained in the usual manner, by taking the curl of the Navier-Stokes equations of viscous fluid flow, and incorporating the Boussinesq approximation of a small perturbation density $\bar{\rho}$. This results in

$$
\frac{\partial \zeta}{\partial t}+u \frac{\partial \zeta}{\partial r}+w \frac{\partial \zeta}{\partial z}-\frac{u \zeta}{r}=\frac{1}{D F^{2}} \frac{\partial \bar{\rho}}{\partial r}+\frac{1}{D \operatorname{Re}}\left(\nabla^{2} \zeta-\frac{\zeta}{r^{2}}\right)
$$

which is related to the vorticity equation given by Batchelor [2, p. 267]. The first term on the right-hand side of equation (5.5) is the rate of change of vorticity due to buoyancy, and is sometimes referred to as the baroclinic component [31, p. 166]. In this equation, the Froude number $F$ and density ratio $D$ are as given in (2.1), and the constant $\operatorname{Re}=\rho_{1} A V / \mu$ is the Reynolds number for the flow, in which $\mu$ is the dynamic viscosity of the fluid.

In this viscous Boussinesq approximation, the density changes continuously in the fluid, and so the sharp interface in Section 2 is replaced with a diffuse interfacial zone 
of rapid but smooth change. In addition, it is necessary in the numerical solution to impose an impenetrable wall at some radius $r=\beta$, sufficiently far from the plume. The boundary layers on the bottom $z=0$ and the artificial wall at $r=\beta$ are assumed to be thin, and so are ignored in this approximation, following Farrow and Hocking [9]. The vertical velocity component $w$ is still assumed to obey the bottom condition (2.3), and the density perturbation along the bottom boundary has the form

$$
\bar{\rho}(r, 0, t)= \begin{cases}-(D-1) & \text { if } 0<r<\beta \\ 0 & \text { if } r>\beta,\end{cases}
$$

corresponding to the two different fluids within the plume and outside it. The appropriate boundary condition on the vertical boundary at $r=\beta$ is

$$
u=0 \quad \text { and } \quad \zeta=0,
$$

since this artificial wall is impenetrable. Tangential slip is permitted at this outer boundary, since the boundary layer there is ignored, and consequently the vorticity is assumed to be zero.

It is convenient to represent the streamfunction and the density perturbations as sums of steady (time independent) terms and unsteady functions, according to the expressions

$$
\bar{\rho}(r, z, t)=\bar{\rho}_{S}(r)+\bar{\rho}_{U}(r, z, t), \quad \Psi(r, z, t)=\Psi_{S}(r)+\Psi_{U}(r, z, t) .
$$

The steady component $\bar{\rho}_{S}$ of the density perturbation must satisfy the bottom condition (5.6) for the density, and so it is required that the unsteady component $\bar{\rho}_{U}$ there should be zero, from (5.7). Equation (5.6) for the steady component of the density perturbation is represented by the Fourier series expression

$$
\bar{\rho}_{S}(r)=\sum_{m=1}^{M} C_{m, 0} \mathrm{~J}_{0}\left(\gamma_{m} r\right),
$$

truncated after the $M$ th Fourier mode, in which the coefficients are

$$
C_{k, 0}=-(D-1) \frac{2 \mathrm{~J}_{1}\left(\gamma_{k}\right)}{\gamma_{k} \beta^{2} \mathrm{~J}_{0}\left(j_{1, k}\right)^{2}} .
$$

In (5.8) and (5.9), the constants $j_{1, k}, k=1, \ldots, M$, represent the first $M$ nonzero roots of the first-kind Bessel function $J_{1}$ of order one, consistently with the notation of Abramowitz and Stegun [1, p. 370], and it is also convenient to define the related constants $\gamma_{k}=j_{1, k} / \beta$. Similarly, the steady component of the vertical velocity $w$ must satisfy the boundary condition (2.3), and therefore has the form

$$
w_{S}(r)= \begin{cases}1 & \text { if } 0<r<1 \\ 0 & \text { if } r>1,\end{cases}
$$


which is expressed using the Fourier series

$$
w_{S}(r)=\sum_{m=1}^{M} B_{m, 0} \mathrm{~J}_{0}\left(\gamma_{m} r\right)
$$

with the coefficients

$$
B_{k, 0}=\frac{2 \mathbf{J}_{1}\left(\gamma_{k}\right)}{\gamma_{k} \beta^{2} \mathrm{~J}_{0}\left(j_{1, k}\right)^{2}} .
$$

Using the streamfunction relations (5.3), the vertical velocity (5.10) can be integrated to give the steady component of the streamfunction,

$$
\Psi_{S}(r)=\sum_{m=1}^{M} \frac{1}{\gamma_{m}} B_{m, 0} \mathrm{~J}_{1}\left(\gamma_{m} r\right)
$$

It is now necessary to express the time-dependent portions of the flow variables by the Fourier series

$$
\begin{aligned}
& \Psi_{U}(r, z, t)=\sum_{m=1}^{M} \sum_{n=1}^{N} B_{m, n}(t) \mathbf{J}_{1}\left(\gamma_{m} r\right) \sin \left(\alpha_{n} z\right), \\
& \bar{\rho}_{U}(r, z, t)=\sum_{m=1}^{M} \sum_{n=1}^{N} C_{m, n}(t) \mathbf{J}_{0}\left(\gamma_{m} r\right) \sin \left(\alpha_{n} z\right)+\sum_{n=1}^{N} C_{0, n}(t) \sin \left(\alpha_{n} z\right),
\end{aligned}
$$

where the $\alpha_{n}$ are the constants defined in equation (3.2). It follows from (5.4) that the vorticity has the form

$$
\zeta(r, z, t)=\sum_{m=1}^{M} \sum_{n=1}^{N} A_{m, n} B_{m, n}(t) \mathbf{J}_{1}\left(\gamma_{m} r\right) \sin \left(\alpha_{n} z\right)+\sum_{m=1}^{M} \gamma_{m} B_{m, 0} \mathbf{J}_{1}\left(\gamma_{m} r\right),
$$

in which the steady-state coefficients $B_{m, 0}$ are as defined in equation (5.11), and the remaining constants are

$$
A_{m, n}=\gamma_{m}^{2}+\alpha_{n}^{2}
$$

These Fourier series (5.12)-(5.14) are now substituted into the governing equations of motion, (5.2) and (5.5), to determine the unknown coefficients $B_{m, n}(t)$ and $C_{m, n}(t)$. The equations are spectrally decomposed by multiplying by the basis functions and integrating over the computational domain $0<r<\beta, 0<z<h$. Applying this procedure to the vorticity equation (5.5) produces the system of differential equations

$$
\begin{aligned}
B_{k, \ell}^{\prime}(t)=- & \frac{4}{h \beta^{2} \mathbf{J}_{2}\left(j_{1, k}\right)^{2} A_{k, \ell}} \int_{0}^{\beta} \int_{0}^{h} G(r, z, t) \sin \left(\alpha_{\ell} z\right) r \mathbf{J}_{1}\left(\gamma_{k} r\right) d z d r \\
& -\frac{2 \gamma_{k}}{h \alpha_{\ell} D F^{2} A_{k, \ell}} C_{k, 0}-\frac{\gamma_{k}}{D F^{2} A_{k, \ell}} C_{k, \ell}(t) \\
& -\frac{2 \gamma_{k}^{3}}{h \alpha_{\ell} D \operatorname{Re} A_{k, \ell}} B_{k, 0}-\frac{A_{k, \ell}}{D \operatorname{Re}} B_{k, \ell}(t),
\end{aligned}
$$


in which $G(r, z, t)=u \partial \zeta / \partial r+w \partial \zeta / \partial z-u \zeta / r$ and the steady-state coefficients $C_{k, 0}$ are as given in equation (5.9). The density transport equation (5.2) is likewise subjected to a similar Fourier analysis, which produces two more sets of differential equations,

$$
\begin{aligned}
C_{k, \ell}^{\prime}(t)=- & \frac{4}{h \beta^{2} \mathrm{~J}_{0}\left(j_{1, k}\right)^{2}} \int_{0}^{h} \int_{0}^{\beta}\left(u \frac{\partial \bar{\rho}}{\partial r}+w \frac{\partial \bar{\rho}}{\partial z}\right) \sin \left(\alpha_{\ell} z\right) r \mathrm{~J}_{0}\left(\gamma_{k} r\right) d r d z \\
& -\frac{2 \sigma \gamma_{k}^{2}}{\alpha_{\ell} h} C_{k, 0}-\sigma A_{k, \ell} C_{k, \ell}(t), \\
C_{0, \ell}^{\prime}(t)=- & \frac{4}{h \beta^{2}} \int_{0}^{h} \int_{0}^{\beta}\left(u \frac{\partial \bar{\rho}}{\partial r}+w \frac{\partial \bar{\rho}}{\partial z}\right) \sin \left(\alpha_{\ell} z\right) r d r d z-\sigma \alpha_{\ell}^{2} C_{0, \ell}(t) .
\end{aligned}
$$

This system (5.15)-(5.17) represents $(2 M+1) N$ ordinary differential equations for the $(2 M+1) N$ Fourier coefficients $B_{m, n}(t), C_{m, n}(t)$ and $C_{0, n}(t)$. These differential equations are integrated forward in time using the classical fourth-order Runge-Kutta method. The integrals expressed in each of these equations are evaluated using composite trapezoidal rule quadrature. The numerical solutions are generally started from the steady-state components of the flow, expressions (5.8) and (5.10), and this requires the initial conditions to be $B_{m, n}(0)=0, C_{m, n}(0)=0$ and $C_{0, n}(0)=0$. We have also experimented with initial conditions taken from the steady asymptotic solutions derived in Section 4. Accurate solutions have been obtained here with $M=N=20$, giving an overall system of $(2 M+1) N=820$ ordinary differential equations for the Fourier coefficients, to be integrated forward in time. The double integrals in these expressions have been evaluated over a grid of $101 \times 201$ mesh points.

\section{Results}

In this section, some results of our numerical computations are illustrated both for the inviscid and the viscous (Boussinesq) plumes. The solutions shown are typical of the formation and evolution of the plume over time, for a wide variety of the physical parameters. For ease of reference, the solutions presented in this section all assume a plume height $h=20$. The numerical quadratures were performed with 201 equally spaced points along the $z$-axis.

Figure 2 presents a comparison of the nonlinear inviscid solutions, computed with the spectral method described in Section 3, with the results of the steady asymptotic solution. In each diagram, interface profiles are shown for the three different dimensionless times $t=5,20$ and 35. The plume was started from a vertical jet with initial radius $R=1$ and unit vertical speed everywhere. As time progresses, a constriction wave moves up the plume, similar to the predictions of the unsteady asymptotic theory. Thus, at the early time $t=5$, much of the initial interface profile is still visible, but eventually the interface is drawn into a shape that narrows as the height $z$ is increased, since Figure 2 does not involve entrainment $(k=0)$. Also shown is the steady-state asymptotic plume radius calculated from equation (4.5), sketched with a dashed line. The density ratio is taken to be $D=1.05$. In Figure 2(a) the 
(a)

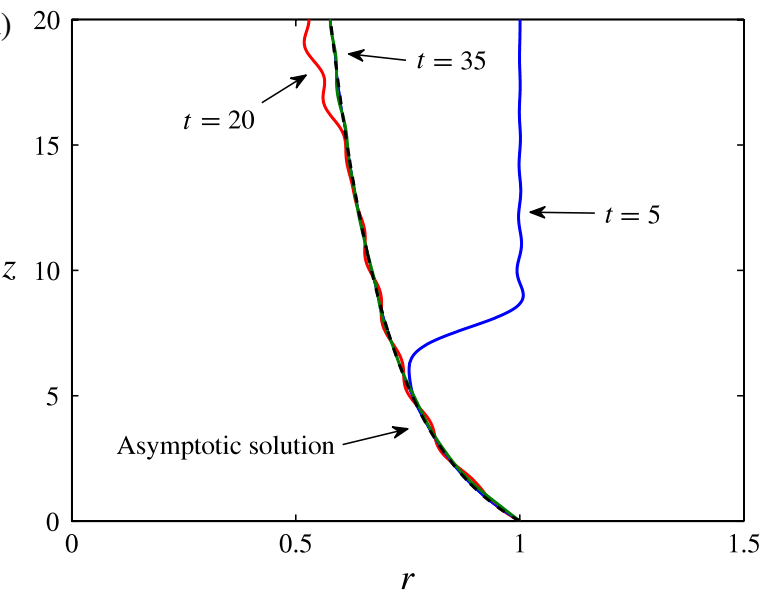

(b)

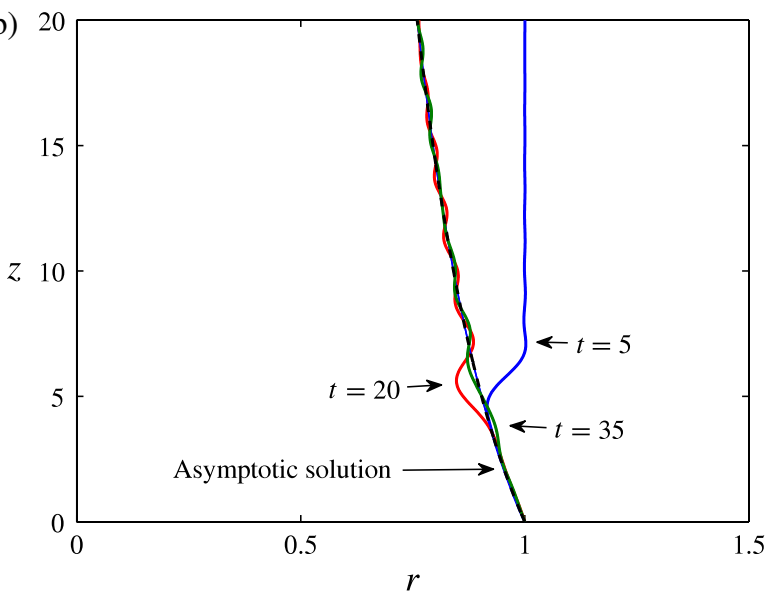

FIGURE 2. Comparison of two inviscid nonentraining $(k=0)$ plume profiles at the three different times $t=5,20$ and 35, with the asymptotic solution (dashed lines). Results are shown for a plume height $h=20$ and density ratio $D=1.05$, for Froude numbers (a) $F=0.5$ and (b) $F=1$.

Froude number is $F=0.5$, representing a relatively slow outflow from the nozzle, and it can be seen that the nonlinear unsteady interface converges slowly to the asymptotic result, as time increases. In the process, it generates a number of small-amplitude unsteady waves, and these continue to move up the plume for some considerable time. For the higher Froude number case $F=1$ illustrated in Figure 2(b), convergence to a steady-state configuration is much more rapid, and by dimensionless time $t=35$ the interface is almost coincident with the asymptotic prediction (4.5). This demonstrates that these round plumes are stable when directed vertically, within the assumptions of the inviscid model, and that the asymptotic solution of Section 4 provides a good approximation to the eventual steady state. 

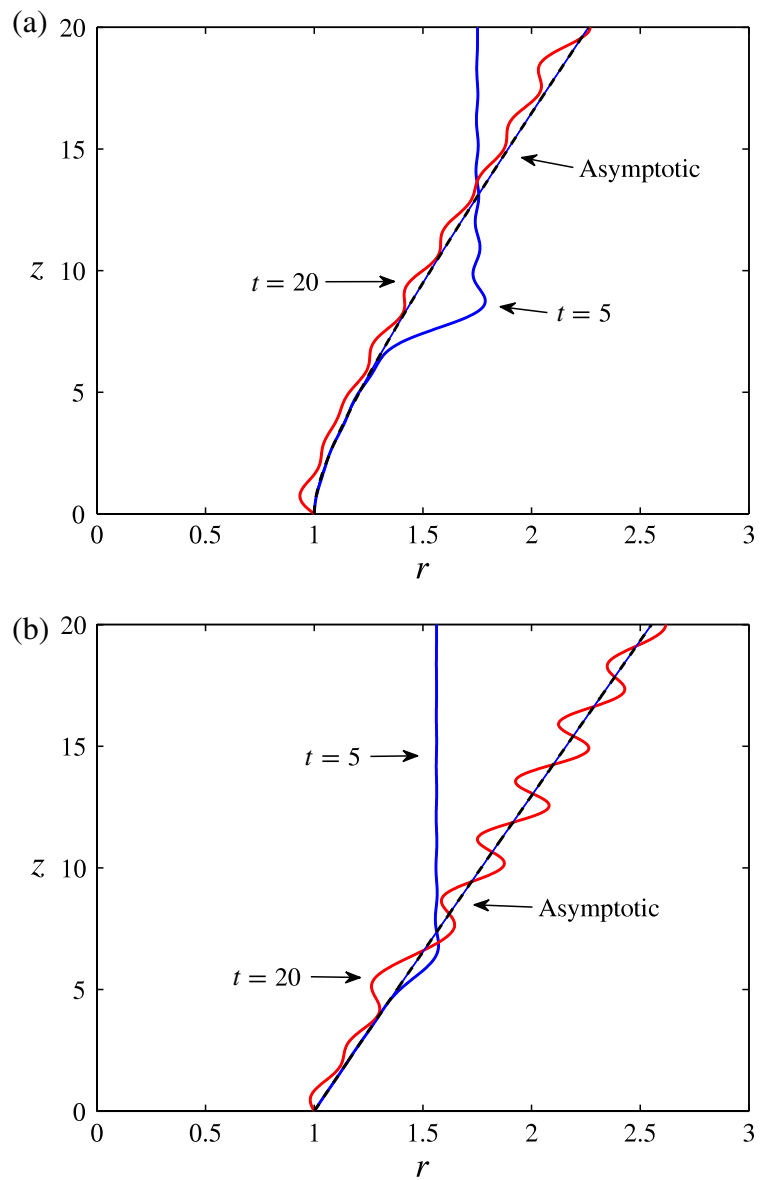

Figure 3. Comparison of two inviscid entraining plume profiles at the two different times $t=5$ and $t=20$, with the asymptotic solution (dashed lines). Results are shown for entrainment coefficient $k=0.1$, plume height $h=20$ and density ratio $D=1.05$, for Froude numbers (a) $F=0.5$ and (b) $F=1$.

In Figure 3, nonlinear inviscid solutions at different times are again presented, although now for a nonturbulent entraining plume with entrainment coefficient $k=0.1$. Solutions with a variety of different values for $k$ have been studied, and all are qualitatively similar to those shown in Figure 3. In addition, the prediction (4.6) for the steady-state interface obtained from the asymptotic solution is also shown, sketched using dashed lines. Two sets of results are shown, for Froude numbers $F=0.5$ and $F=1$ in Figures 3(a) and 3(b), respectively. The nonlinear inviscid interface is shown at the two different times $t=5$ and 20 in each plot, and the solution was again started from a uniform upward flow in a plume of initial radius $R=1$. Once again, it may be seen that the plume begins to develop as a constriction wave that moves upwards with time, although entrainment of the ambient fluid in this case eventually causes 


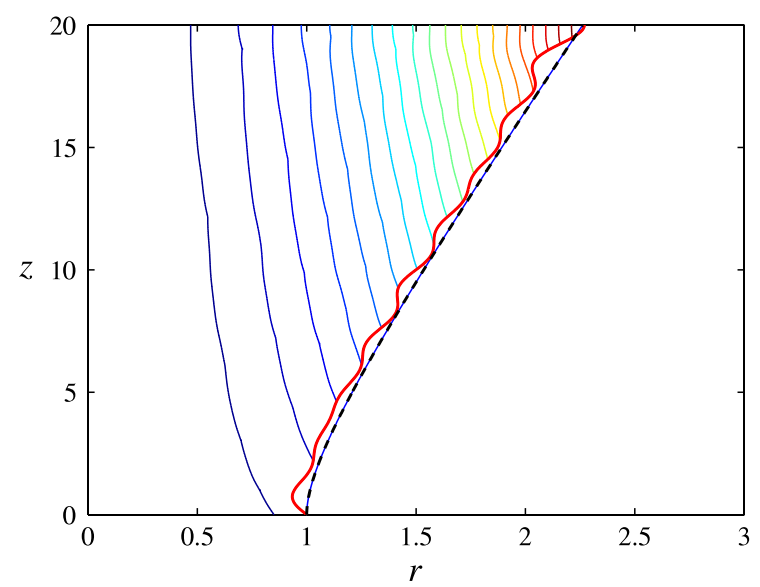

FIGURE 4. Streamlines for the inviscid entraining plume at time $t=20$, for Froude number $F=0.5$ and $h=20, D=1.05$. The entrainment coefficient is $k=0.1$, and the interface is also shown, as in Figure 3(a). The steady asymptotic approximation is shown with a dashed line.

the plume to widen with height, and at later times a series of small unsteady waves propagate upwards.

In the absence of entrainment in the inviscid model, there is no flow normal to the interface, at least once steady state has been achieved. By an examination of the continuity equation (5.1), it may be shown that, for steady flow, the contours of the function $r \Psi$ are streamlines, directed tangentially to the fluid velocity vector. Consequently, when the streamlines are plotted for the nonentraining inviscid plume at steady state, they are all parallel to the interface $r=R$, since no fluid can cross that boundary. By contrast, in the entraining case, when streamlines are plotted as contours of the function $r \Psi$, they are no longer parallel to the interface at steady state, as a consequence of the approximate nonturbulent entrainment boundary condition developed in equation (2.6). This is illustrated in Figure 4. Here the entrainment coefficient is $k=0.1$ and the Froude number is $F=0.5$, as in Figure 3(a), and the solution is displayed at time $t=20$. The interface and its asymptotic approximation (4.6) at steady state are shown, as previously. In addition, the streamlines within the plume are displayed, and they highlight the way in which the outer ambient fluid is entrained in through the boundary and then moves almost vertically within the plume. Similar results are obtained with a variety of values of the entrainment coefficient $k$, although it is observed that, for $k$ sufficiently large, the curvature at the interface may become large at certain points, and the numerical scheme then fails to continue to larger times. In those cases, it appears that the inviscid interface might form a finite-time curvature singularity similar to that discovered by Moore [20] and encountered under some circumstances for planar plumes by Forbes and Hocking [12].

The rise of a viscous plume is shown in Figure 5. This solution was started from a plume of unit width, with unit vertical motion in its interior, and allowed to evolve 


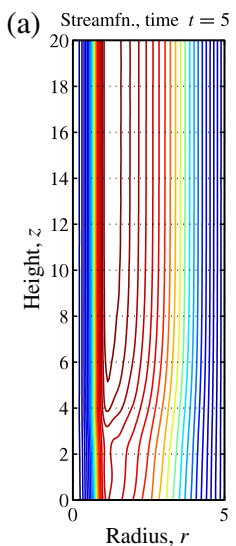

(a)

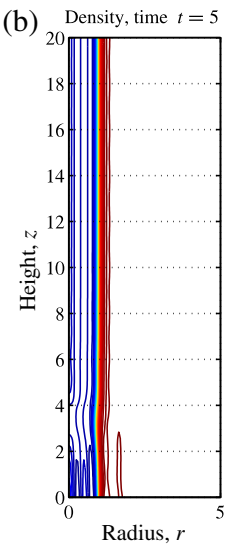

(c) Vorticity, time $t=5$

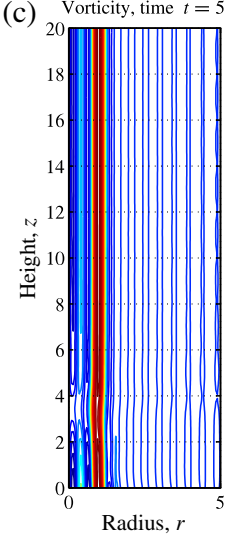

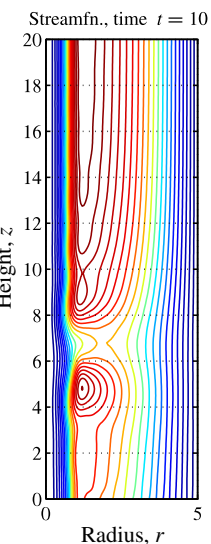
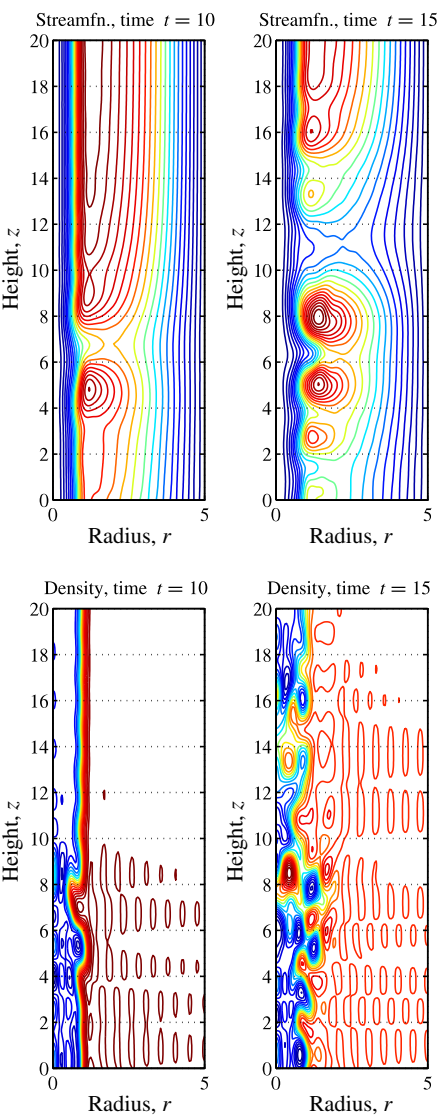

Vorticity, time $t=10$

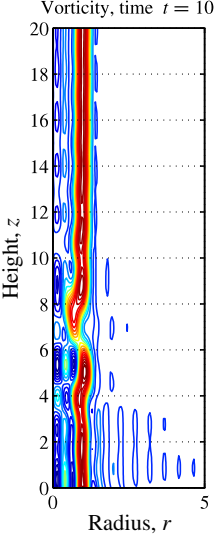

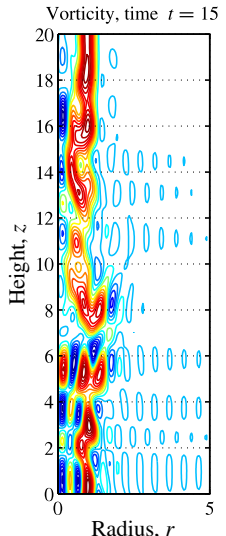

FIGURE 5. Solutions for a viscous plume at the three different times $t=5,10$ and 15 , with Reynolds number $\operatorname{Re}=1000$ and Froude number $F=1$. The density ratio is $D=1.05$ and the diffusion coefficient is $\sigma=10^{-3}$. This solution was started from uniform flow in a plume of unit width, and results are presented as contours of (a) $r \Psi$ (the streamlines), (b) density $\bar{\rho}$ and (c) vorticity $\zeta$. 
forward in time using the algorithm of Section 5. The Reynolds number was taken to be $\operatorname{Re}=1000$, and the Froude number $F=1$. Streamlines are shown in Figure 5(a) at the three times $t=5,10$ and 15. Initially, no vortices are present in the flow, but as time progresses, an entrainment vortex is formed, and may be seen in the solution at time $t=10$ at about height $z=4$. Similar behaviour was observed for the planar plume by Forbes and Hocking [12]. At the later time $t=15$, further entrainment vortices are formed in the interfacial zone near the plume, but at about this time the presence of the "artificial" wall at $r=\beta$ begins to exert an influence, so that results beyond this time are not considered.

Density contours for this same plume are presented in Figure 5(b), at the same three times. At early times there is a clear interfacial zone with a rapid but smooth change of density, at about radius $r \approx 1$, consistently with the Boussinesq approximation, but as time progresses, entrainment vortices are generated and the effect of mixing becomes evident. Figure 5(c) shows contours of the vorticity at these three times, and confirms that, at early times, the flow is essentially irrotational almost everywhere, except in the narrow region of the interfacial zone, where vorticity is generated by viscous shear. At the later time $t=15$, the interfacial zone breaks down through viscous diffusion and convection, so that substantial mixing takes place.

As a means of assessing the effect of viscous entrainment, the streamlines are shown in Figure 6, for the same parameters as in Figure 5 but at the time $t=12$. Vortex generation is evident at several sites up the plume. Overlaid on this figure is the asymptotic solution (4.5) in the case of no entrainment $(k=0)$, for the same Froude number $F=1$ and density ratio $D=1.05$. In the absence of entrainment, equation (4.4) predicts that the fluid in the plume continues to move faster as it rises, so that, by conservation of mass, the plume necessarily becomes increasingly narrow with height $z$, as can be seen in Figure 6. By including a small degree of inviscid entrainment, $k>0$, it is possible to make the overall shape of the asymptotic plume conform a little more closely to the viscous result, at certain times, but it is nevertheless clear that there is no real equivalence between the approximate nonturbulent entrainment modelled by equation (2.6) and the more complex processes of vortex generation at the interface that occur in the genuinely viscous case.

To reinforce the fact that this is an axisymmetric plume in three dimensions, as opposed to the planar geometry considered by Forbes and Hocking [12], the density contour $\bar{\rho}=-0.02$ in the interfacial zone has been extracted and rotated about the $z$ axis. This gives an indication of the actual plume shape, and is presented in Figure 7, for the same time $t=12$ as in Figure 6. The effect of plume constriction due to the presence of entrainment vortices is evident, and there are even overhanging sections along the interface. The plume widens again near the top, due to viscous entrainment of the ambient fluid.

\section{Conclusion}

This paper has considered the evolution of an axisymmetric vertical plume in a fully nonlinear inviscid model of the phenomenon. Attention has been focused on 


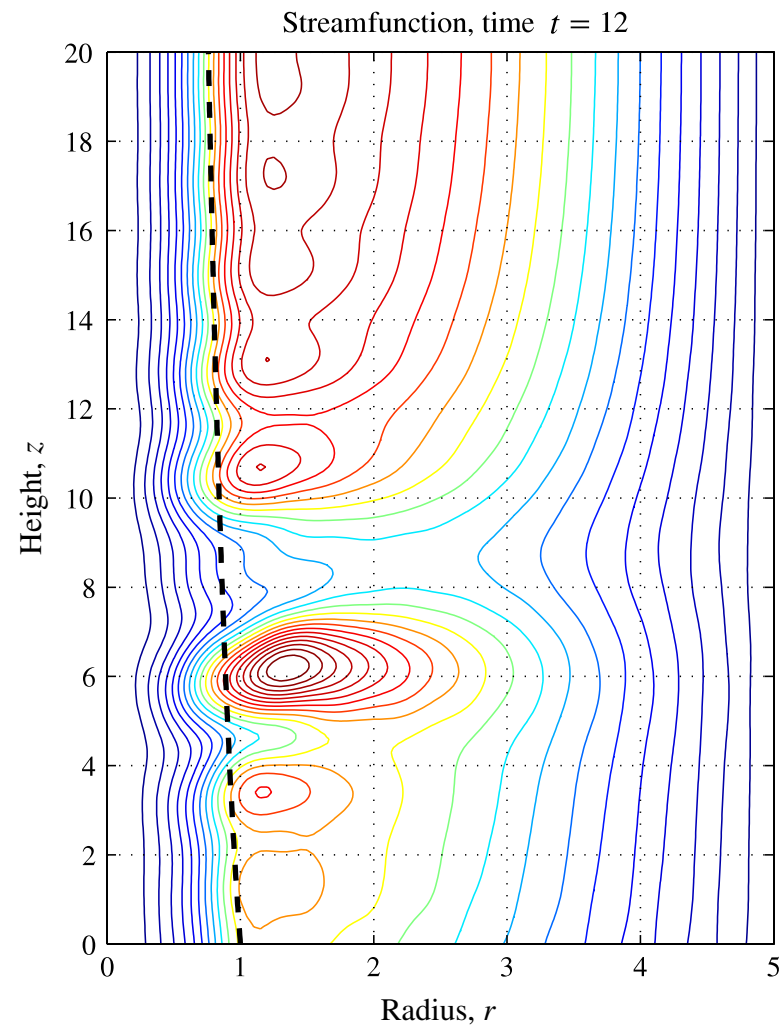

FIGURE 6. Streamlines for the viscous plume in Figure 5, with Reynolds number Re $=1000$, at time $t=12$. The dashed line indicates the asymptotic interface in the nonentraining case $k=0$.

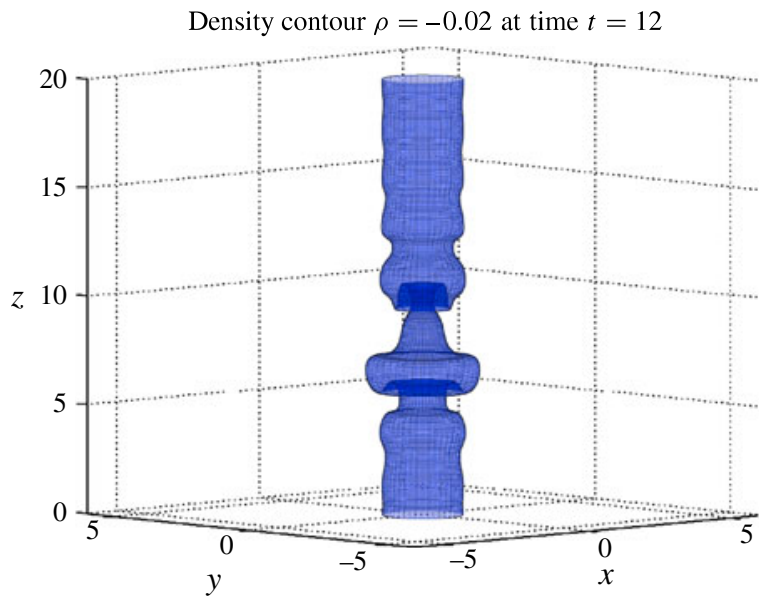

FIGURE 7. The density contour $\bar{\rho}=-0.02$ for the viscous plume in Figure 5 , with Reynolds number $\operatorname{Re}=1000$, at time $t=12$. 
the approximate transitional parameter region $F \sim 1$ and $200<\operatorname{Re}<1000$, rather than the fully turbulent situation modelled in the "classical" MTT plume theory [21]. The results of Forbes and Hocking [12] for inviscid planar plumes have been generalized to the case of a round plume directed vertically, and it has been established that, within the assumptions of inviscid theory, the plume is stable and converges to a steadystate profile for large times. That profile is accurately predicted by an asymptotic theory based on an assumption that the inviscid plume is slender. For completeness, surface-tension effects were also included in these inviscid results, originally as a means of regularizing the numerically computed plume profiles if these had proven to be unstable, as was the case for planar plumes directed nonvertically in the work of Forbes and Hocking [12]. However, as instability was not a feature of the vertical axisymmetric inviscid plumes encountered here, the inclusion of surface tension proved to be unnecessary and it had a negligible effect on the plume shapes.

An approximate entrainment model was also proposed for these transitional, nonturbulent plumes, adapted from the classical MTT plume model [21] in the turbulent case. The underlying premise is that the vortex sheet present at the interface of the fluid plume is responsible for entraining some of the outer ambient fluid with a velocity normal to the interface that is proportional to the tangential speed of the fluid within the plume. This approximate entrainment condition in equation (2.6) generalizes the classical kinematic condition which requires no fluid flow normal to the free surface, and can be implemented within an otherwise inviscid flow model. The asymptotic slender-plume theory was again applied to this situation, and the prediction (4.6) it makes for the interface shape is qualitatively in agreement with profiles in the paper by Hunt and Kaye [16], for example. In addition, the nonlinear numerical results for this case confirm that these predicted interface profiles are stable within the assumptions of inviscid theory, for these vertical plumes.

Viscous solutions have also been computed, under the Boussinesq approximation, again extending the similar calculations of Forbes and Hocking [12] for planar plumes to this axisymmetric three-dimensional geometry. A full spectral method has been used since it has the advantage over finite-difference techniques, such as that used by Chen and Forbes [6], that the Poisson equation (5.4) for the streamfunction is solved trivially, without the need for iteration. The viscous results are coarsely in agreement with the predictions of the inviscid entrainment model (2.6) developed here; when entrainment coefficients of the order $k \approx 0.1$ are chosen, both the viscous and inviscid cases suggest an overall widening of the plume as height from the inlet nozzle increases. Nevertheless, our inviscid entrainment model has only limited capacity to mimic true viscous entrainment, for these transitional plumes. This is because the approximate condition (2.6) contains no mechanism for the generation of vorticity at the interface, whereas in the true viscous case there are vortices formed at several locations up the plume. We have experimented with viscous plumes started from several different initial configurations, but all develop axisymmetric vortices ringing the round plume, at various different heights.

Obtaining the viscous solutions presented in this paper represents a significant computational exercise. Nevertheless, it is possible to extend these results to cases 
in which the plume rises at an angle, although that would destroy the axisymmetric geometry assumed here, so incurring a far greater computational cost. In the planar case, Forbes and Hocking [12] found that nonvertical plumes were subject to a type of lateral instability, and it is likely that a similar phenomenon occurs in the fully threedimensional case also. It is of interest to know in more detail how the jet or plume develops from its initial injection at the nozzle, and this will be considered in future work.

\section{Acknowledgements}

Constructive criticism by three anonymous reviewers is gratefully acknowledged.

\section{References}

[1] M. Abramowitz and I. A. Stegun (eds), Handbook of mathematical functions: with formulas, graphs and mathematical tables (Dover Publications, New York, 1972.

[2] G. K. Batchelor, An introduction to fluid dynamics (Cambridge University Press, Cambridge, 1967).

[3] G. S. Bhat and A. Krothapalli, "Simulation of a round jet and a plume in a regional atmospheric model", Monthly Weather Rev. 128 (2000) 4108-4117; doi:10.1175/1520-0493(2000)129<4108:SOARJA>2.0.CO;2.

[4] M. S. Borgas and E. O. Tuck, "Thin slender water jets", J. Fluid Mech. 118 (1982) 379-391; doi:10.1017/S0022112082001128.

[5] E. C. Button, J. F. Davidson, G. J. Jameson and J. E. Sader, "Water bells formed on the underside of a horizontal plate. Part 2. Theory", J. Fluid Mech. 649 (2010) 45-68; doi:10.1017/S0022112009993363.

[6] M. J. Chen and L. K. Forbes, "Accurate methods for computing inviscid and viscous KelvinHelmholtz instability", J. Comput. Phys. 230 (2011) 1499-1515; doi:10.1016/j.jcp.2010.11.017.

[7] P Christodoulides and F. Dias, "Impact of a rising stream on a horizontal plate of finite extent", J. Fluid Mech. 621 (2009) 243-258; doi:10.1017/S0022112008004746.

[8] O. M. Faltinsen, O. F. Rognebakke, I. A. Lukovsky and A. N. Timokha, "Multidimensional modal analysis of nonlinear sloshing in a rectangular tank with finite water depth", J. Fluid Mech. 407 (2000) 201-234; doi:10.1017/S0022112099007569.

[9] D. E. Farrow and G. C. Hocking, "A numerical model for withdrawal from a two-layer fluid", J. Fluid Mech. 549 (2006) 141-157; doi:10.1017/S0022112005007561.

[10] L. K. Forbes, "The Rayleigh-Taylor instability for inviscid and viscous fluids", J. Engrg. Math. 65 (2009) 273-290; doi:10.1007/s10665-009-9288-9.

[11] L. K. Forbes, M. J. Chen and C. E. Trenham, "Computing unstable periodic waves at the interface of two inviscid fluids in uniform vertical flow", J. Comput. Phys. 221 (2007) 269-287; doi:10.1016/j.jcp.2006.06.010.

[12] L. K. Forbes and G. C. Hocking, "Unsteady plumes in planar flow of viscous and inviscid fluids", IMA J. Appl. Math. to appear; doi:10.1093/imamat/hxr045.

[13] J. F. Geer and J. C. Strikwerda, "Vertical slender jets with surface tension", J. Fluid Mech. 135 (1983) 155-169; doi:10.1017/S0022112083003006.

[14] G. C. Hocking and L. K. Forbes, "Steady flow of a buoyant plume into a constant-density layer", J. Engrg. Math 67 (2010) 341-350; doi:10.1007/s10665-009-9324-9.

[15] G. R. Hunt and T. S. van den Bremer, "Classical plume theory: 1937-2010 and beyond", IMA J. Appl. Math. 76 (2011) 424-448; doi:10.1093/imamat/hxq056.

[16] G. R. Hunt and N. B. Kaye, "Lazy plumes", J. Fluid Mech. 533 (2005) 329-338; doi: $10.1017 /$ S002211200500457X. 
[17] E. Kaminski, S. Tait and G. Carazzo, "Turbulent entrainment in jets with arbitrary buoyancy", J. Fluid Mech. 526 (2005) 361-376; doi:10.1017/S0022112004003209.

[18] W. Lin and S. W. Armfield, "Onset of entrainment in transitional round fountains", Int. J. Heat Mass Transfer 51 (2008) 5226-5237; doi:10.1016/j.ijheatmasstransfer.2008.02.047.

[19] E. J. List, "Turbulent jets and plumes", Annu. Rev. Fluid Mech. 14 (1982) 189-212; doi:10.1146/annurev.fl.14.010182.001201.

[20] D. W. Moore, "The spontaneous appearance of a singularity in the shape of an evolving vortex sheet", Proc. R. Soc. Lond. A 365 (1979) 105-119; doi:10.1098/rspa.1979.0009.

[21] B. R. Morton, G. Taylor and J. S. Turner, "Turbulent gravitational convection from maintained and instantaneous sources", Proc. R. Soc. Lond. A 234 (1956) 1-23; doi:10.1098/rspa.1956.0011.

[22] F. Plourde, M. V. Pham, S. D. Kim and S. Balachandar, "Direct numerical simulations of a rapidly expanding thermal plume: structure and entrainment interaction", J. Fluid Mech. 604 (2008) 99-123; doi:10.1017/S0022112008001006.

[23] G. Proskurowski, M. D. Lilley, D. S. Kelley and E. J. Olson, "Low temperature volatile production at the Lost City Hydrothermal Field, evidence from a hydrogen stable isotope geothermometer", Chem. Geol. 229 (2006) 331-343; doi:10.1016/j.chemgeo.2005.11.005.

[24] C. S. Reynolds, S. Heinz and M. C. Begelman, "The hydrodynamics of dead radio galaxies", Mon. Not. R. Astron. Soc. 332 (2002) 271-282; doi:10.1046/j.1365-8711.2002.04724.x.

[25] M. M. Scase, A. J. Aspden and C. P. Caulfield, "The effect of sudden source buoyancy flux increases on turbulent plumes", J. Fluid Mech. 635 (2009) 137-169; doi:10.1017/S002211200900740X.

[26] M. M. Scase and R. E. Hewitt, "Unsteady turbulent plume models", J. Fluid Mech. 697 (2012) 455-480; doi:10.1017/jfm.2012.77.

[27] J. J. Stoker, Water waves (Wiley Interscience, New York, 1957).

[28] E. O. Tuck, "Annular water jets", IMA J. Appl. Math. 29 (1982) 45-58; doi:10.1093/imamat/29.1.45.

[29] J. S. Turner, "Buoyant plumes and thermals", Annu. Rev. Fluid Mech. 1 (1969) 29-44; doi:10.1146/annurev.fl.01.010169.000333.

[30] J. S. Turner, "Turbulent entrainment: the development of the entrainment assumption, and its applications to geophysical flows", J. Fluid Mech. 173 (1986) 431-471; doi:10.1017/S0022112086001222.

[31] G. K. Vallis, Atmospheric and oceanic fluid dynamics: fundamentals and large-scale circulation (Cambridge University Press, Cambridge, 2006).

[32] W. S. D. Wilcock, "Cellular convection models of mid-ocean ridge hydrothermal circulation and the temperatures of black smoker fluids", J. Geophys. Res. 103 (1998) 2585-2596; doi:10.1029/97JB03252.

[33] A. W. Woods, "Turbulent plumes in nature", Annu. Rev. Fluid Mech. 42 (2010) 391-412; doi:10.1146/annurev-fluid-121108-145430. 